\title{
Autophagy inhibition enhances radiosensitivity of Eca-109 cells via the mitochondrial apoptosis pathway
}

\author{
HUA TAO, PUDONG QIAN, JINCHENG LU, YESONG GUO, HUANFENG ZHU and FEIJIANG WANG \\ Department of Radiotherapy Oncology, Jiangsu Cancer Hospital, Jiangsu Institute of Cancer Research, \\ Nanjing Medical University Affiliated Cancer Hospital, Nanjing, Jiangsu 210009, P.R. China
}

Received October 26, 2017; Accepted March 26, 2018

DOI: 10.3892/ijo.2018.4349

\begin{abstract}
Autophagy inhibition is crucial for the improvement of the efficacy of radiotherapy in cancer. The aim of the present study was to determine the potential therapeutic value of autophagy and its correlation with mitochondria in human esophageal carcinoma cells following treatment with ionizing radiation (IR). Autophagy in Eca-109 cells was induced under poor nutrient conditions. The formation of autophagic vacuoles was monitored using electron microscopy. In addition, cell apoptosis after IR and mitochondrial membrane potential (MMP) were analyzed by flow cytometry. LC3, beclin-1, cytochrome $c$ and apoptosis-related proteins were assayed by western blotting. A nude mouse xenograft model was also employed to verify the biological effects and mechanisms underlying autophagy in vivo. The formed autophagic vesicles and increased LC3 II/LC3 I ratio indicated marked induction of autophagy by Earle's balanced salt solution (EBSS) in Eca-109 cells. 3-Methyladenine or LY294002 significantly antagonized EBSS-induced autophagy and increased apoptosis of irradiated cells, suggesting that autophagy inhibition conferred radiosensitivity in vitro. Notably, IR induced prominent release of cytochrome $c$ and Bax activation, and decreased Bcl-2 and MMP expression in Eca-109 cells under poor nutrient conditions. Of note, these changes were more prominent following pretreatment with autophagy inhibitors. In vivo, IR treatment mildly delayed tumor growth, but the radiotherapeutic effect was improved significantly by abolishing autophagy. Furthermore, mitochondrial signaling was investigated in the Eca-109 xenograft nude mice model, and
\end{abstract}

Correspondence to: Dr Feijiang Wang, Department of Radiotherapy Oncology, Jiangsu Cancer Hospital, Jiangsu Institute of Cancer Research, Nanjing Medical University Affiliated Cancer Hospital, 42 Baiziting Road, Nanjing, Jiangsu 210009, P.R. China

E-mail: wangfeijiang1964@yeah.net

Abbreviations: EBSS, Earle's balanced salt solution; IR, ionizing radiation; MMP, mitochondrial membrane potential; $\mathrm{SCC}$, squamous cell carcinoma

Key words: squamous cell carcinoma, autophagy, ionizing radiation, radiosensitivity, mitochondria the results were consistent with the in vitro study. Therefore, the mitochondrial pathway may be associated with improvement of radiosensitivity in Eca-109 cells.

\section{Introduction}

Esophageal carcinoma is the eighth most common type of cancer globally $(1,2)$. Squamous cell carcinoma (SCC) is the predominant histological type of esophageal carcinoma (3), but its incidence varies widely by region (4). High morbidity of esophageal SCC has been reported in northern and central China, Turkey, Kazakhstan and northeastern Iran (4,5). Although radiotherapy is key to the treatment of esophageal cancer, sustained remission and long-term survival remain moderate.

Autophagy is a natural homeostatic mechanism that enables cells to maintain energy homeostasis by recycling cellular components in response to nutrient deprivation and stress $(6,7)$. The association between radioresistance and autophagy has been previously investigated (8). Radiotherapy is crucial for the management of unresectable esophageal carcinoma, but radioresistance of the tumor is a hindrance to achieving sustained remission or long-term survival. Autophagy has been reported to enhance the radioresistance of non-small-cell lung cancer (9-11). Preliminary studies indicated that autophagy inhibition improved the radiosensitivity of breast cancer and esophageal SCC, consequently enhancing the cytotoxicity of radiotherapy $(12,13)$. The aim of the present study was to elucidate the potential therapeutic value of autophagy and its underlying mechanism in esophageal SCC.

As the most common mechanism of deregulated cell death in cancer, the mitochondrial pathway acts as 'an enemy within' for cancer treatment (14). Cytochrome $c$, as one of mitochondrial intermembrane space proteins released from damaged mitochondria, is crucial for apoptosis (15). In cervical cancer cells, mitochondria were reported to participate in ionizing radiation (IR)-induced autophagic cell death (16), but their role in esophageal SCC has not been fully elucidated.

Ample evidence suggests that interference with autophagy of cancer cells may increase their sensitivity to treatment, indicating that the promotion of protective autophagy may be a potential mechanism of radiotherapy resistance in cancer (17-21). However, existing studies are not sufficient to confirm the role of autophagy and the detailed underlying mechanisms in esophageal SCC following treatment with IR. 
Therefore, further investigation into the role of autophagy in esophageal SCC may determine whether antagonizing strategies can help improve the outcome of radiotherapy.

\section{Materials and methods}

Reagents and antibodies. RPMI-1640 medium was purchased from Gibco; Thermo Fisher Scientific (Grand Island, NY, USA). The autophagy inhibitors 3-methyladenine (3-MA) and LY294002 were obtained from Sigma-Aldrich; Merck KGaA (St. Louis, MO, USA). Rabbit anti-human/mouse/ rat polyclonal antibody LC3 (E18-5402, 1:500), GAPDH (E1C604, 1:2,000), cytochrome oxidase (COX) IV (E12327, 1:1,000), cleaved caspase-3 (E11-0104L, 1:500), cleaved caspase-8 (E18-5267, 1:1,000), cleaved caspase-9 (E18-5240, $1: 1,000)$, mouse anti-human/mouse monoclonal antibody Bcl-2 (E10-30077, 1:1,000), rabbit anti-human/mouse polyclonal antibody Bax (E11-0773B, 1:500), Beclin-1 (E90562, 1:500), mouse anti-human/mouse/rat monoclonal antibody cytochrome $c$ (E12-378, 1:2000), goat anti-rabbit (E0L3012), goat anti-mouse IgG secondary antibodies (E0L3032), Cell Counting Kit-8 (CCK-8) and cell apoptosis analysis kit were all purchased from EnoGene (New York, NY, USA). Mouse anti-human p53 polyclonal antibody was obtained from BD Pharmingen (554294; San Jose, CA, USA). TRIzol reagent was obtained from Life Technologies; Thermo Fisher Scientific (Carlsbad, CA, USA). RevertAid First Strand cDNA Synthesis kit and QuantiFast SYBR Green PCR kit were purchased from Thermo Fisher Scientific (Waltham, MA, USA). Mitochondrial membrane potential assay kit with JC-1 was obtained from Beyotime Biotechology (Shanghai, China).

Cell culture. The human esophageal carcinoma cell line Eca-109 was acquired from the American Type Culture Collection (Manassas, VA, USA). The cells were cultured in RPMI-1640 medium supplement with $10 \%$ fetal bovine serum (ScienCell, San Diego, CA, USA), 2 mmol/l L-glutamine, $100 \mathrm{U} / \mathrm{ml}$ penicillin and $100 \mu \mathrm{g} / \mathrm{ml}$ streptomycin. All cells were maintained at $37^{\circ} \mathrm{C}$ under a humidified, $5 \% \mathrm{CO}_{2}$ atmosphere.

Animals. Athymic nude mice (BALB/c-nu, female), aged 4-5 weeks, were purchased from Nanjing Model Animal Research Center (animal permission no., SYXK2012-0049). All experiments involving mice were performed in conformity with the guidelines on animal care and experiments on laboratory animals of the Center of Experimental Animals, Nanjing University of Technology (Nanjing, China), and were approved by the Ethics Committee for animal experimentation.

Autophagy and IR. Eca-109 cells were pretreated with the Earle's balanced salt solution (EBSS; $\mathrm{NaCl} 116.36 \mathrm{mM}$, $\mathrm{KCl} 5.37 \mathrm{mM}, \mathrm{CaCl}_{2} 1.80 \mathrm{mM}, \mathrm{MgSO}_{4} \cdot 7 \mathrm{H}_{2} \mathrm{O} 0.81 \mathrm{mM}$, $\mathrm{Na}_{2} \mathrm{HPO}_{4} \cdot 12 \mathrm{H}_{2} \mathrm{O} 6.40 \mathrm{mM}, \mathrm{Na}_{2} \mathrm{HCO}_{3} 26.19 \mathrm{mM}$, and glucose $5.55 \mathrm{mM}$ ), in order to create poor nutrient conditions and induce autophagosome formation. For in vitro radiation experiments, following EBSS treatment, Eca-109 cells were exposed to room temperature and irradiated with a Cobalt-60 radiotherapy apparatus (Theratron $780 \mathrm{c}$; Best Theratronics Ltd., Ottawa, ON, Canada) at the indicated doses. Following irradiation, cell cultures were placed in the cell culture incubator and maintained at $37^{\circ} \mathrm{C}$ under $5 \% \mathrm{CO}_{2}$. Control cells were removed from the cell incubator and placed under the IR source without radiation exposure for the same period. In the combined treatment studies, indicated concentrations of 3-MA or LY294002 were added into the medium prior to irradiation. Cells were further collected for apoptosis analysis and measurement of the relative protein and mRNA expression.

Electron microscopy. Following EBSS treatment, Eca-109 cells were harvested by trypsinization and fixed with $2.5 \%$ glutaraldehyde for at least $24 \mathrm{~h}$. The cells were stained with osmium-thiocarbohydrazide-osmium. Subsequently, the cells were dehydrated in a series of graded ethanol concentrations (70-100\%) and were immersed serially in 1:1 hexamethyldisilazane followed by absolute ethanol. Thin sections $(1-\mu \mathrm{m})$ were cut, and the gels were coated with $500 \AA$ of gold in a JEOL vacuum sputter coater and viewed under a JEOL T300 electron microscope with a scanning attachment (JEOL, Tokyo, Japan).

Cell viability and apoptosis assay. For the cell viability assay, 10,000 cells per well were seeded into a 96-well plate and cultured overnight. Following treatment with 3-MA or LY294002, $10 \mu$ 1 CCK-8 solution was added to each well and the optical density 450 absorbance was measured by a multifunctional microplate reader (Thermo Fisher Scientific) after a 2-h incubation at $37^{\circ} \mathrm{C}$. For the cell apoptosis assay, the cells were stained with Annexin V/propidium iodide (PI), and cell apoptosis was analyzed using flow cytometry. In accordance with the instruction of cell apoptosis analysis kit (EnoGene), cells $\left(5 \times 10^{5}\right)$ were harvested and centrifuged at $1,000 \mathrm{x} \mathrm{g}$ for $5 \mathrm{~min}$. Cell samples were then resuspended in $500 \mu \mathrm{l}$ of binding buffer. Annexin V-enhanced green fluorescent protein (5 $\mu \mathrm{l})$ and PI $(5 \mu \mathrm{l})$ were added to the samples and incubated in the dark for $15 \mathrm{~min}$. A total of $1 \times 10^{4}$ cells were collected per sample and detected on FACSCalibur (BD Biosciences, San Jose, CA, USA). The data were finally analyzed using FlowJo software (FlowJo LLC, Ashland, OR, USA).

Reverse transcription-polymerase chain reaction (RT-PCR) analysis. Cells and tumor tissues were used to investigate the expression of LC3 II and p53 mRNA. In accordance with the manufacturer's protocol, total RNA was isolated with TRIzol reagent (Life Technologies; Thermo Fisher Scientific), and was reverse-transcribed to cDNA using a RevertAid First Strand cDNA Synthesis kit (Thermo Fisher Scientific). The cDNA obtained was amplified using the QuantiFast SYBR Green PCR kit. The assays were performed on an ABI 7500 Fast RealTime PCR system (Applied Biosystems, Waltham, MA, USA). Specific primers were synthesized by Invitrogen and the sequences are listed as follows: GAPDH, forward 5'-CCTCTG ACTTCAACAGCGACAC-3' andreverse 5'-CTGTTGCTGTAG CCAAATTCGT-3'; LC3 II, forward 5'-CAGGTTCACAGAAC CCGCC-3' and reverse 5'-GGTTGCGCTTCACAACTCAG-3'; and p53, forward 5'-TTCGACATAGTGTGGTGG TGC-3' and reverse 5'-GCTGTTCCGTCCCAGTAGATT-3'. Relative gene expression levels were calculated by the $2^{-\Delta \Delta C q}$ method and normalized to GAPDH expression as internal control (22).

Subcellular fractionation. Cells or tumor tissues were homogenized using a Dounce homogenizer in an isotonic buffer 
A

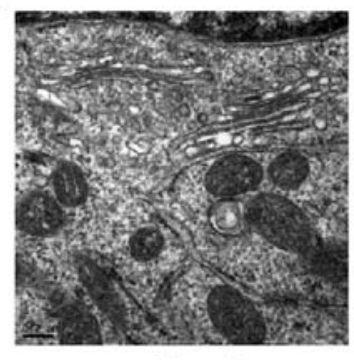

Control

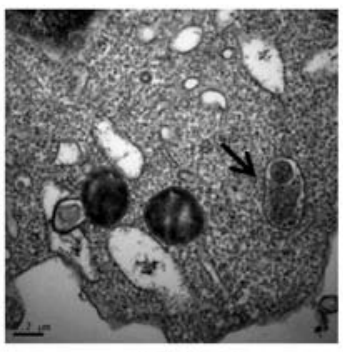

EBSS (8 h)

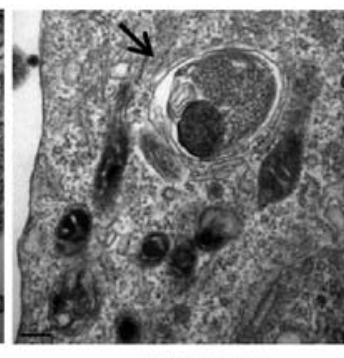

EBSS (12 h)

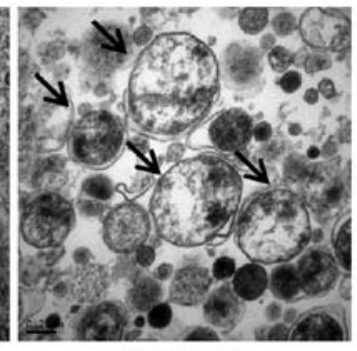

EBSS (24 h)
B

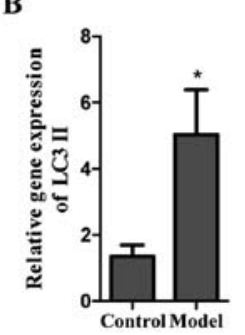

C

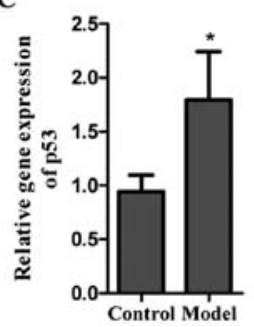

D

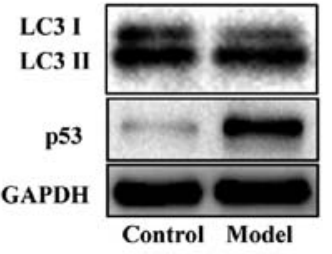

E

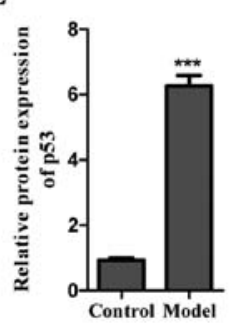

F

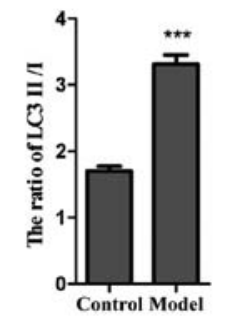

Figure 1. Induction of autophagy by Earle's balanced salt solution (EBSS) in Eca-109 cells. (A) Electron micrographs showed the ultrastructure of Eca-109 cells treated with EBSS at 8, 12 and $24 \mathrm{~h}$, respectively. Autophagic vacuoles were observed after 8 and $12 \mathrm{~h}$ of EBSS treatment, and empty vacuoles (black arrows) were observed after $24 \mathrm{~h}$ of EBSS treatment (scale bar, $0.2 \mu \mathrm{M}$ ). (B and C) At $12 \mathrm{~h}$ after EBSS treatment, the expression of LC3 II and P53 mRNA in the cells was detected by quantitative polymerase chain reaction analysis and (D-F) cell lysates were prepared for immunoblotting for LC3 I, LC3 II and p53. The results of densitometric quantification are shown using GAPDH for normalization (mean \pm standard deviation, $\mathrm{n}=3$, ${ }^{*} \mathrm{P}<0.05$ and ${ }^{* * * *} \mathrm{P}<0.001$ vs. control).

(250 mM sucrose, $1 \mathrm{mM}$ EDTA, $50 \mathrm{mM}$ Tris-HCl, $1 \mathrm{mM}$ DTT, $1 \mathrm{mM}$ PMSF, $1 \mathrm{mM}$ benzamidine, $0.28 \mu / \mathrm{ml}$ aprotinin, $50 \mu \mathrm{g} / \mathrm{ml}$ leupeptin, and $7 \mu \mathrm{g} / \mathrm{ml}$ pepstain), and then centrifuged at $1,000 \mathrm{x} \mathrm{g}$ for $10 \mathrm{~min}$. The resulting supernatant was centrifuged at $10,000 \mathrm{x}$ g for $20 \mathrm{~min}$ and the pellet was collected as the crude mitochondrial fraction. The remaining supernatant was centrifuged at $100,000 \mathrm{x} g$ for $1 \mathrm{~h}$ and the supernatant was collected as the cytosolic fraction. The preparation was used for the following immunoblotting assay.

Western blotting. Western blot analysis was performed according to standard methods. Total cellular and tissue extracts or mitochondrial fraction were prepared by RIPA buffer (Beyotime Institute of Biotechnology, Shanghai, China) according to the manufacturer's instructions. Protein concentrations were determined using the Bradford assay. Equal amounts of protein (30-50 $\mu \mathrm{g}$, depending on the protein) were separated by SDS-PAGE and transferred onto PVDF membranes. The membranes were incubated with the indicated primary antibodies and then horseradish peroxidase-conjugated secondary antibodies. Protein bands were developed using an ECL detection system. The grey density of target bands was analyzed by ImageJ software (National Institutes of Health, Bethesda, MD, USA) and normalized to GAPDH or the mitochondrial marker COX IV.

Measurement of mitochondrial membrane potential (MMP). The MMP of Eca-109 cells was measured using the cationic dye JC-1 (Beyotime Institute of Biotechnology). After treatment, Eca-109 cells were incubated with $\mathrm{JC}-1$ at $37^{\circ} \mathrm{C}$ and $5 \% \mathrm{CO}_{2}$ for $20 \mathrm{~min}$. JC-1 monomers emit green fluorescence spontaneously, whereas they emit red fluorescence upon entering the mitochondria of normal cells. Thus, the ratio of red to green reflects the value of MMP.-
Animal xenograft analysis. Eca-109 cells $\left(5 \times 10^{6}\right)$, in exponential growth phase were injected subcutaneously into the right axilla of nude mice to grow as tumor xenografts. When the tumor volume reached $100 \mathrm{~mm}^{3}$, tumor-bearing mice were randomly assigned to four treatment groups ( $n=6$ per group) as follows: Control, IR alone (8 Gy), 3-MA alone (10 mg/kg intraperitoneally every other day for 2 weeks), and IR combined with 3-MA. Mice from the IR group received localized tumor radiation with 8 Gy using a Cobalt 60 radiotherapy apparatus (Theratron 780c; Best Theratronics Ltd.). 3-MA was administrated intraperitoneally every other day for 2 weeks until the end of the experiment. The control group received no IR. Tumor size was determined every other day by measuring the tumor diameter with calipers. Tumor volume was calculated using the following formula: Volume $=$ width $^{2} \mathrm{x}$ length $/ 2$. The mice were sacrificed when the tumors exceeded $20-25 \%$ of the body mass or when the tumor volume reached 1,000-1,500 $\mathrm{mm}^{3}$. The tumors were excised and weighed for analysis.

Statistical analysis. Data are expressed as means \pm standard deviation. Multiple groups were compared with one-way analysis of variance and two groups with Dunnett's test, using GraphPad Prism 5 (GraphPad Software, San Diego, CA, USA). Statistical significance was set at $\mathrm{P}<0.05$.

\section{Results}

Induction of autophagy by EBSS. To evaluate the potential of EBSS on autophagy induction, Eca-109 cells were examined at different time points after being cultured in EBSS (8, 12 and $24 \mathrm{~h}$ ). Electron microscopy was used to determine the subcellular changes following exposure to EBSS. As shown in Fig. 1A, no autophagic vesicles were observed in untreated Eca-109 cells, in contrast with those cultured in EBSS for 8, 
A
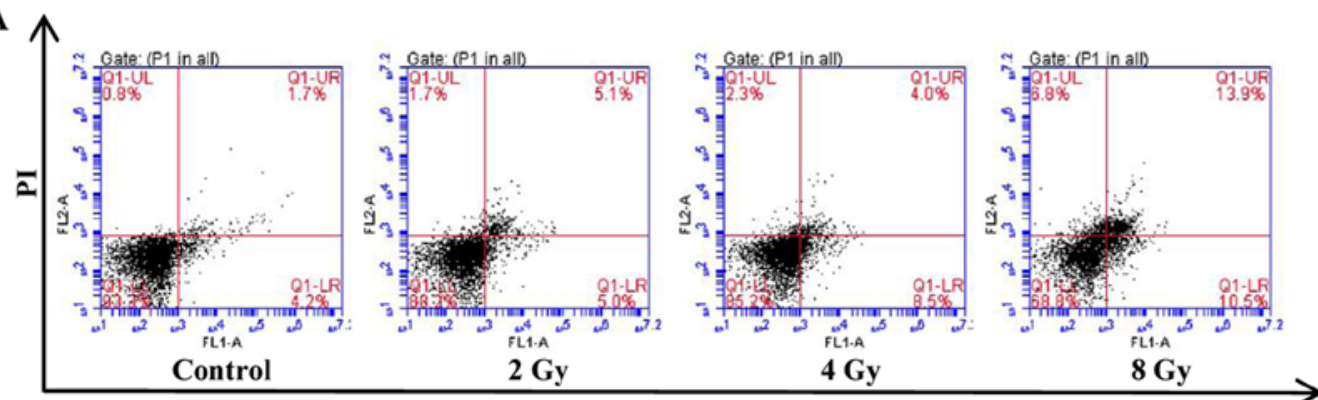

Annexin V-EGFP
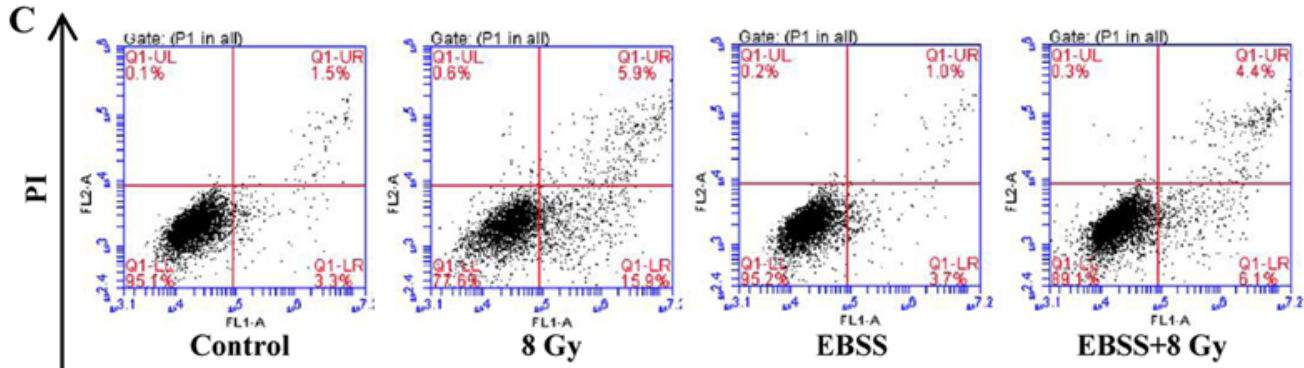

Annexin V-EGFP
B

D
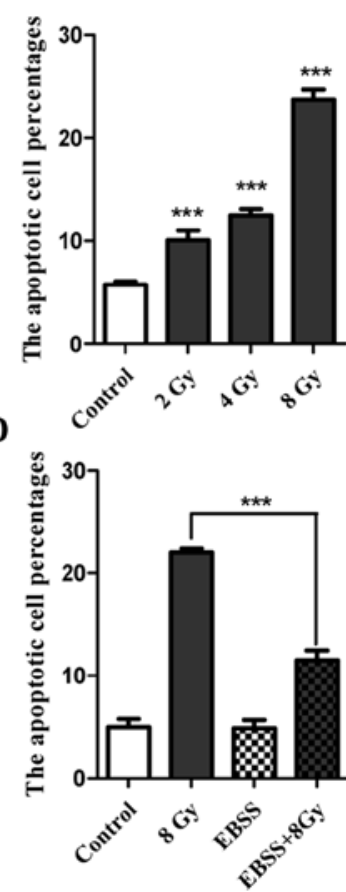

Figure 2. Apoptosis analysis of Eca-109 cells following irradiation with or without EBSS treatment. (A and B) Eca-109 cells were irradiated at different doses and subsequently cultured for $12 \mathrm{~h}$, and the apoptosis of the cells was assessed by flow cytometry (mean \pm standard deviation, $\mathrm{n}=3$, ${ }^{* * *} \mathrm{P}<0.001 \mathrm{vs}$. control) (C and D) Eca-109 cells were first cultured in EBSS for $12 \mathrm{~h}$ to induce autophagy, and further irradiated with 8 Gy prior to another 12 -h culture. Cell apoptosis was determined and compared with that in cells that were only irradiated (mean \pm standard deviation, $\mathrm{n}=3$, $^{* * *} \mathrm{P}<0.001$ ). EBSS, Earle's balanced salt solution; PI, propidium iodide; EGFP, enhanced green fluorescent protein.

12 and $24 \mathrm{~h}$. Compared with $8 \mathrm{~h}$, the vesicles formed after $12 \mathrm{~h}$ of treatment with EBSS were more remarkable, with lamellar structures and residual digested material. However, only several empty vacuoles were observed in the cells at $24 \mathrm{~h}$. In addition, the protein and gene levels of LC3 II were detected in cells cultured in EBSS for $12 \mathrm{~h}$, in which LC3 was used as a specific autophagy marker. The data demonstrated that poor nutrient conditions provided by EBSS markedly decreased LC3 I, but increased LC3 II, as demonstrated by the increased ratio of LC3 II/LC3 I. Of note, a similar trend was observed for p53 (Fig. 1B-F). The observation of the subcellular structure and analysis of LC3 and p53 formation suggested that EBSS-induced autophagy led to no significant cell apoptosis until $12 \mathrm{~h}$. Therefore, the process of autophagy was examined post-EBSS treatment for $12 \mathrm{~h}$ in subsequent experiments.

Radiosensitivity of Eca-109 cells and attenuation effect of autophagy. Cell apoptosis induced by IR was determined in Eca-109 cells. Following exposure to IR at the indicated dose, cell apoptosis was assayed by flow cytometry. The results indicated that the number of apoptotic cells increased in a dose-dependent manner (Fig. 2A and B). To determine whether autophagy could attenuate radiosensitivity, the apoptosis rate of cells that were treated with IR under starvation conditions was compared with that of cells that were only irradiated or starved. As shown in Fig. 2C and D, 12 h of EBSS treatment did not noticeably increase cell apoptosis compared with untreated cells, but significantly weakened the potential of apoptosis induction when combined with IR (8 Gy). Collectively, these findings demonstrated that the radiosensitivity of Eca-109 cells may be attenuated by starvation-induced autophagy.

LY294002 or 3-MA alone inhibit autophagy without induction of cell apoptosis within a certain concentration range. The viability and apoptosis of cells exposed to EBSS with different concentrations of LY294002 (Fig. 3A, C and E) or 3-MA (Fig. 3B, D and F) pretreatment were analyzed. The results indicated that autophagy inhibitors LY294002 and 3-MA did not significantly inhibit cell viability or induce cell apoptosis until the concentrations were increased to $20 \mu \mathrm{M}$ for LY294002 and $2 \mathrm{mM}$ for 3-MA. The autophagy of cells that were pretreated with LY294002 $(10 \mu \mathrm{M})$ and 3-MA $(1 \mathrm{mM})$ under EBSS starvation conditions was also evaluated by analyzing LC3 and p53. As shown in Fig. 4A-E, LY294002 at $10 \mu \mathrm{M}$ not only decreased the gene expression of LC3 II and $\mathrm{p} 53$, but also significantly reduced the protein expression ratio of LC3 II and LC3. Furthermore, despite being increased in EBSS-treated cells, p53 was also decreased by LY294002 at $10 \mu \mathrm{M}$. A similar trend was detected for 3-MA at $1 \mathrm{mM}$ (Fig. 4F-J). Collectively, these results indicate that autophagy inhibitors LY294002 and 3-MA at a suitable concentration may significantly inhibit autophagy without causing extensive cell death.

Inhibition of autophagy increases the radiosensitivity of Eca-109 cells in vitro. To investigate the role of autophagy inhibitors in the radiosensitivity of Eca-109 cells, the apoptosis rate of cells irradiated at 8 Gy was detected with or without autophagy inhibitors. As a result, treatment with 8 Gy alone 
A

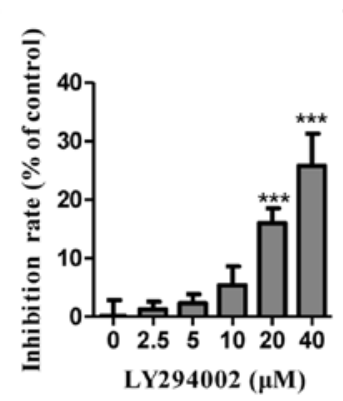

B

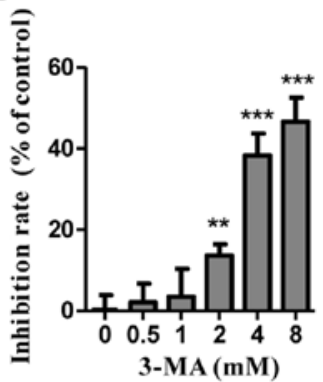

C

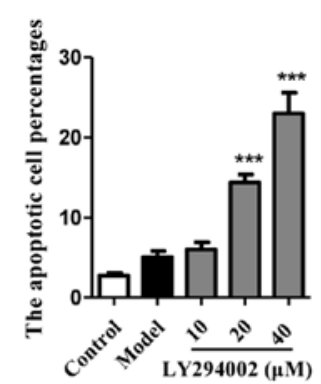

D

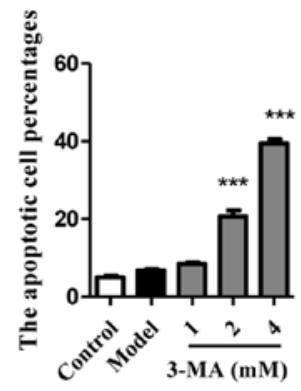

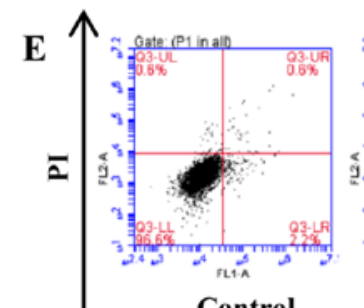

Control

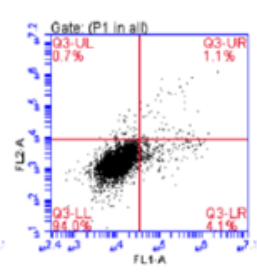

Model

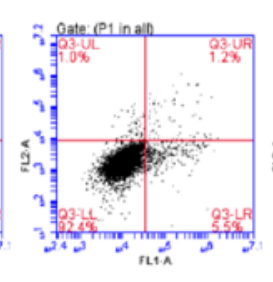

$10 \mu \mathrm{M}$

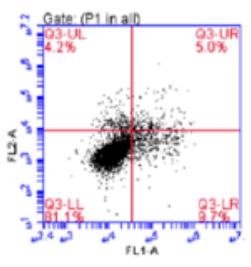

$20 \mu \mathrm{M}$

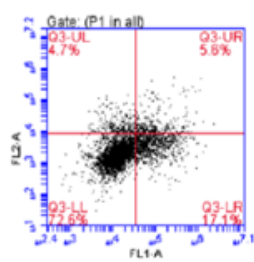

$40 \mu \mathrm{M}$

Annexin V-EGFP
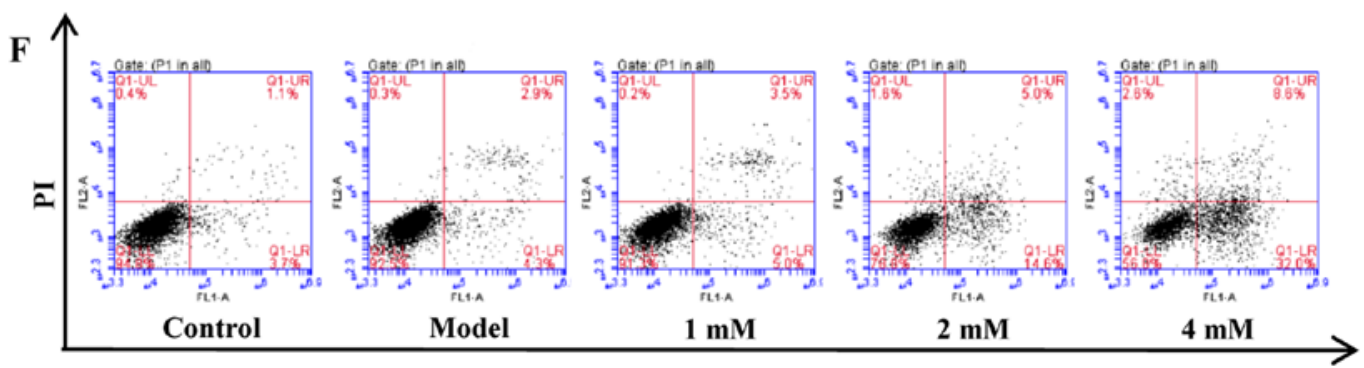

Annexin V-EGFP

Figure 3. Effect of autophagy inhibitors LY294002 and 3-MA on viability and apoptosis of Eca-109 cells following EBSS autophagy induction. Before replacing the culture medium with EBSS, LY294002 and 3-MA at the indicated concentrations were added into the medium. After 5 min, the culture medium was replaced with EBSS containing LY294002 or 3-MA. After $12 \mathrm{~h}$, cell viability and apoptosis-inhibiting ability of LY294002 and 3-MA were evaluated by the Cell Counting Kit-8 assay (A and B) and flow cytometry (C-F) (mean \pm standard deviation, $n=3,{ }^{* *} \mathrm{P}<0.01$ and ${ }^{* * *} \mathrm{P}<0.001$ vs. control). EBSS, Earle's balanced salt solution; 3-MA, 3-methyladenine; PI, propidium iodide; EGFP, enhanced green fluorescent protein.
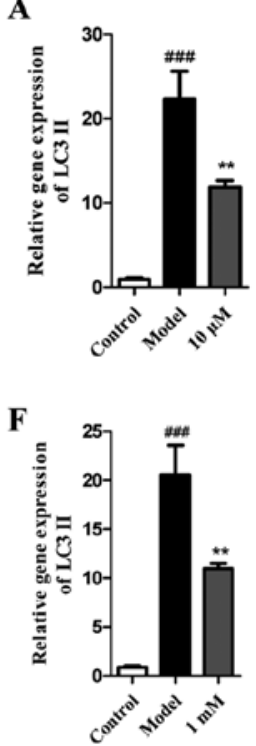

B
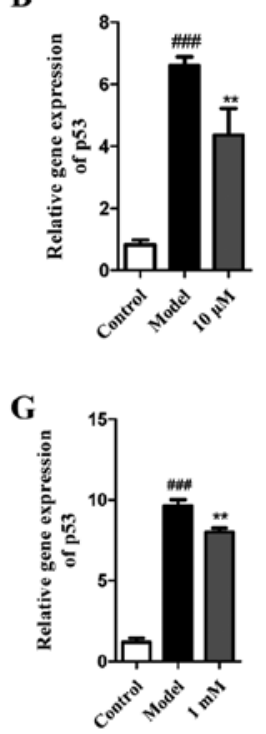

C

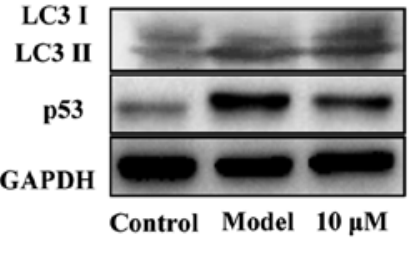

$\mathbf{H}$

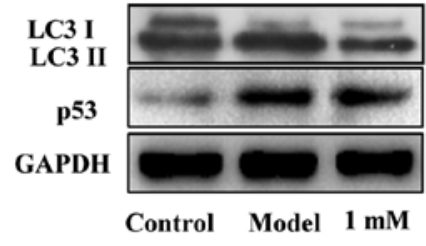

D

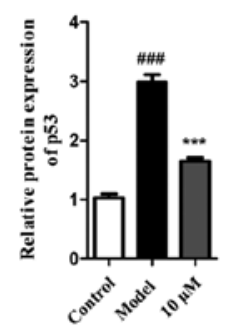

I

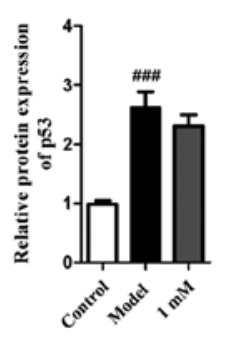

$\mathbf{E}$

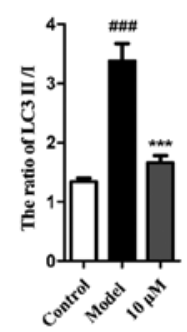

$\mathbf{J}$

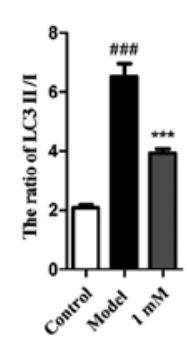

Figure 4. LY294002 and 3-MA downregulated the expression of LC3 and p53 at the mRNA and protein level under autophagy-inducing conditions. At $12 \mathrm{~h}$ after autophagy induced by EBSS with or without autophagy inhibitor pretreatment (10 $\mu \mathrm{M}$ for LY294002 and $1 \mathrm{mM}$ for 3-MA), cell lysates were prepared from Eca-109 cells to analyze the expression of LC3 II and p53 by quantitative polymerase chain reaction analysis (A and B, and F-G) and immunoblotting (C-E and H-J). The results of densitometric quantification were shown using GAPDH for normalization ( $m e a n \pm \operatorname{standard}$ deviation, $\mathrm{n}=3$, ${ }^{\# \#} \mathrm{P}<0.001 \mathrm{vs}$. control, ${ }^{* *} \mathrm{P}<0.01 \mathrm{vs}$. model). All analyses performed in this study are representative of three independent experiments. 3-MA, 3-methyladenine; EBSS, Earle's balanced salt solution. 
A

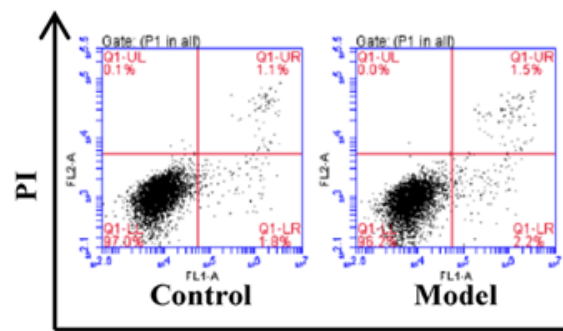

C

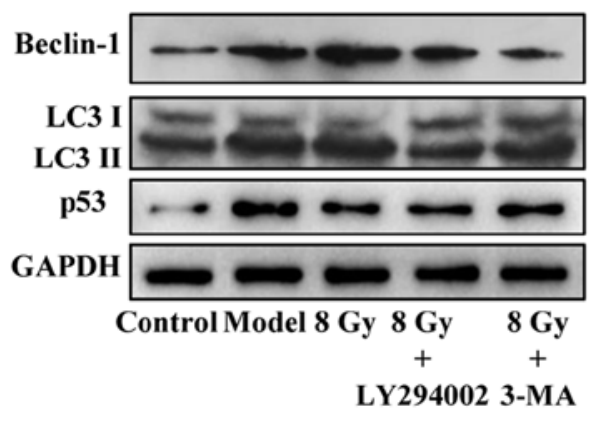

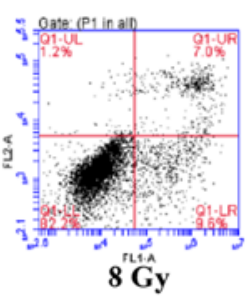

Annexin V-EGFP
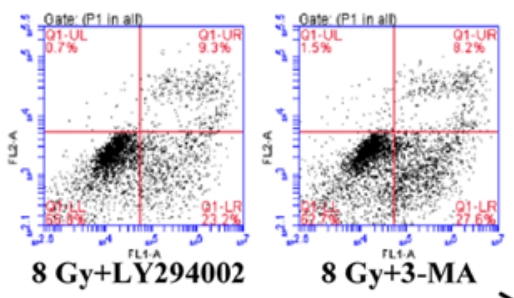

$\longrightarrow$

D

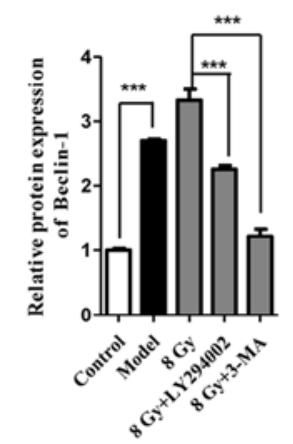

B

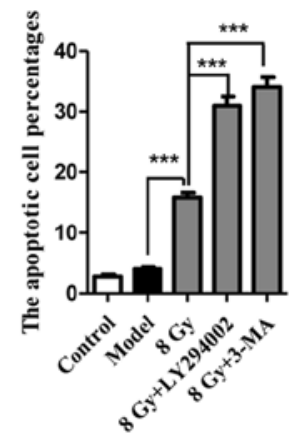

F

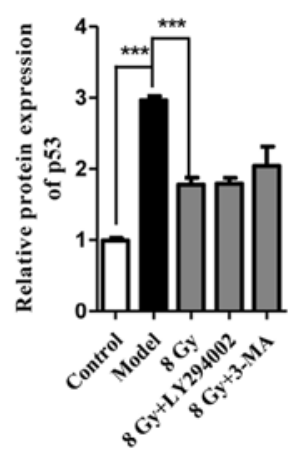

Figure 5. Effect of LY294002 and 3-MA on the radiosensitivity of Eca-109 cells. (A and B) Cell apoptosis was analyzed 12 h after treatment with IR (8 Gy) with or without pretreatment with autophagy inhibitors $\left(10 \mu \mathrm{M}\right.$ for LY294002 and $1 \mathrm{mM}$ for 3-MA). (mean \pm standard deviation, $\mathrm{n}=3$, $\left.^{* * *} \mathrm{P}<0.001\right)$. After treatment as indicated, Eca-109 cells were analyzed for the expression of Beclin-1, LC3 and p53 (mean \pm standard deviation, $\mathrm{n}=3$, ${ }^{* *} \mathrm{P}<0.01$ and $\left.{ }^{* * *} \mathrm{P}<0.001\right) .3-\mathrm{MA}$, 3-methyladenine; IR, ionizing radiation; PI, propidium iodide; EGFP, enhanced green fluorescent protein.

led to a reduced percentage of surviving cells compared with that in the sham group. Furthermore, the apoptosis-induced potential of IR was markedly enhanced following pretreatment with LY294002 and 3-MA (Fig. 5A and B). In addition, detection of beclin-1, LC3 and p53 suggested that LY294002 and 3-MA accelerated cell death and inhibited cell autophagy concurrently (Fig. 5C-F). In summary, these results demonstrated that autophagy inhibition exhibited a radiosensitivity potential in vitro.

Mitochondria are involved in enhancement of radiosensitivity in Eca-109 cells induced by autophagy inhibition. Mitochondria are closely associated with cell death. To assess the changes and the role of mitochondria following radiation and/or autophagy inhibition, apoptosis-related proteins, cytochrome $c$ and MMP were examined. As shown in Fig. 6C, following IR together with EBSS treatment, increased expression of activated caspase- 3 , caspase- 8 and caspase- 9 and decreased levels of Bcl-2 were observed in Eca-109 cells. We also observed increased accumulation of Bax in mitochondria and a large release of cytochrome $c$ into the cytosolic fraction (Fig. 6A and B). Compared with IR alone, the changes in cytochrome $c, \mathrm{Bcl}-2$ and Bax became more prominent following combination with autophagy inhibitors (Fig. 6A-C). As regards MMP, it was observed that radiation (8 Gy) prominently reduced red fluorescence but enhanced green fluorescence. This trend was further enhanced by autophagy inhibitors (Fig. 6D and E), suggesting that MMP was reduced in Eca-109 cells. These results revealed that autophagy inhibition increased the radiosensitivity of
Eca-109 cells, potentially through the mitochondrial apoptotic pathway.

Combination of autophagy inhibitor and IR suppresses the tumorigenesis of Eca-109 cells in a nude mouse xenograft model. Since the additive effects of autophagy inhibition, particularly by 3-MA, on the radiosensitity of Eca-109 cells has been established, a nude mouse xenograft model was utilized to validate the biological effects and underlying mechanisms by in vivo 3-MA administration. Cell suspensions were injected subcutaneously into the right axilla of athymic nude mice. The mice were then randomly divided into the four indicated groups, which were treated with DMSO (vehicle of 3-MA), radiation (8 Gy), 3-MA $(10 \mathrm{mg} / \mathrm{kg})$, and radiation together with 3-MA. As shown in Fig. 7A-C, tumor volume and weight were measured and the data suggested that the tumor size in the model animals markedly enlarged compared with baseline. IR treatment slightly delayed tumor growth. Furthermore, tumor growth was significantly delayed when the animals were treated with IR and 3-MA, whereas treatment with 3-MA alone did not exert a noticeable effect on tumor growth compared with the model group. The immunoblotting analysis revealed that IR treatment promoted the release of cytochrome $c$ and Bax activation, along with decreased levels of Bcl-2. Consistent with the autophagy inhibition effect in vitro, this effect of IR treatment was markedly potentiated by abolishing autophagy (Fig. 7D-F). In summary, these results suggested that combination treatment with 3-MA and IR enhanced the response of esophageal carcinoma cells to radiotherapy in vivo. 
A

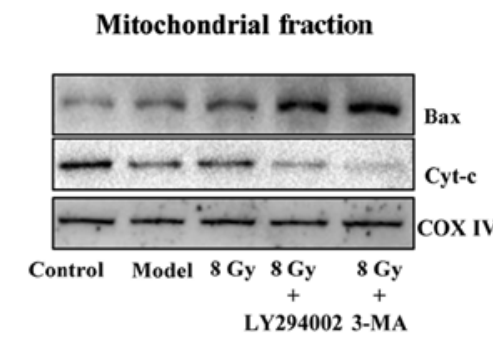

B

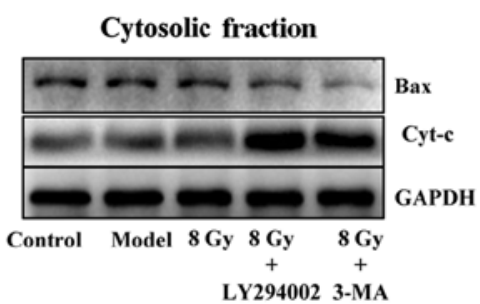

C

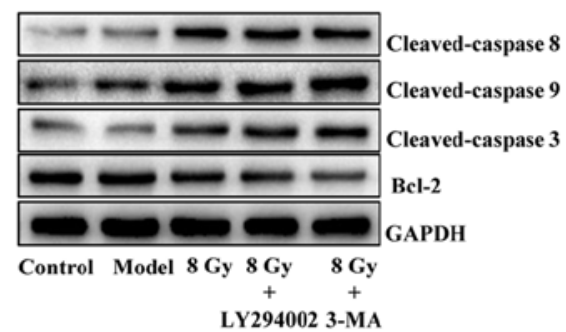

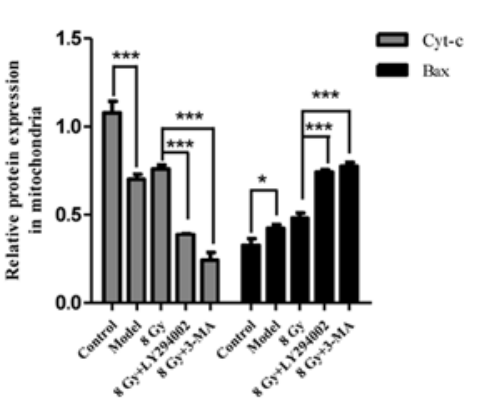

D

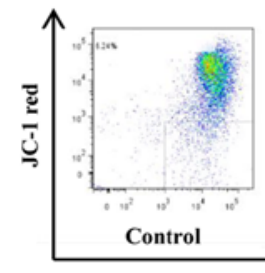

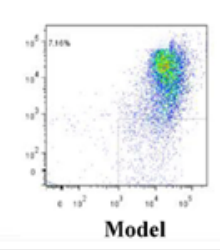
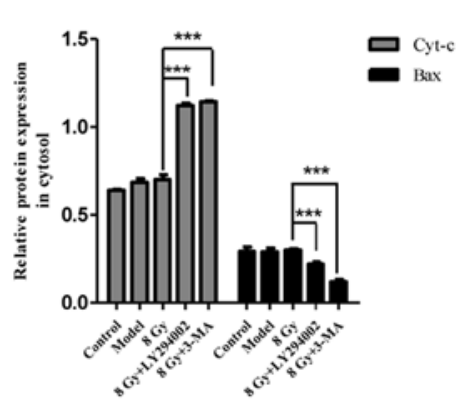

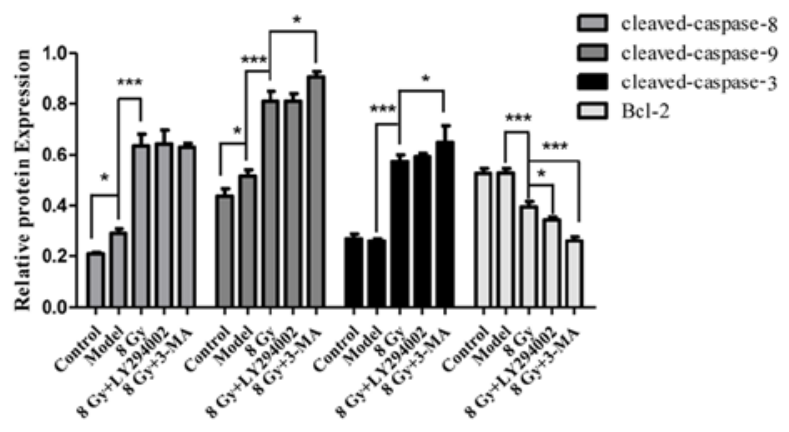

$\mathbf{E}$
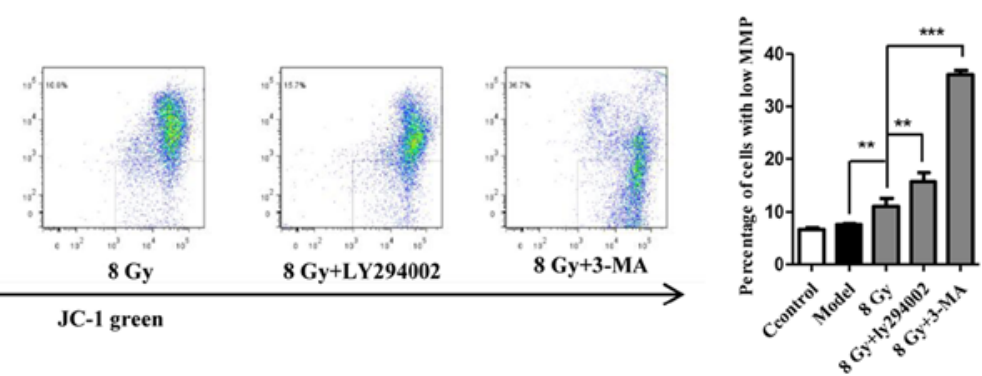

Figure 6. Involvement of mitochondrial apoptotic pathway in radiosensitivity after autophagy inhibition in Eca-109 cells. At $12 \mathrm{~h}$ after treatment with IR (8 Gy) with or without pretreatment with autophagy inhibitors (10 $\mu \mathrm{M}$ for LY294002 and $1 \mathrm{mM}$ for 3-MA), cytochrome $c$ release (A) and Bax translocation to mitochondria (B), and the cellular apoptosis initiators caspase- 8 and caspase- 9 , the effector caspase-3, and the apoptotic protein Bcl-2 (C) in Eca-109 cells were analyzed by western blotting. GAPDH and COX IV were used as internal protein loading controls for the cytosolic and mitochondrial fractions, respectively. (D and E) After treatment as in A, representative flow cytometric analysis of JC-1 assay was conducted, and the depolarized cells exhibited decreased red fluorescence and enhanced green fluorescence. The histogram presents the change of green fluorescence intensity in Eca-109 cells after various treatments (mean \pm standard deviation, $\mathrm{n}=3,{ }^{*} \mathrm{P}<0.05,{ }^{* *} \mathrm{P}<0.01$ and ${ }^{* * *} \mathrm{P}<0.001$ ). COX, cytochrome oxidase; IR, ionizing radiation; 3-MA, 3-methyladenine.

\section{Discussion}

Autophagy enables cells to degrade intracellular molecules to support cell survival, and it has historically been observed in cells under nutrient deprivation conditions (6). As a characteristic of autophagy, the formation of autophagic vacuoles encompasses cellular components to be digested via fusion with lysosomes (23). In autophagy, organelles and proteins to be degraded are sequestered into autophagosomes. During its formation, the cytosolic isoform LC3 I is conjugated to phosphatidylamine to form LC3 II. LC3 II is indispensable for the expansion of autophagosomal membrane. Furthermore, as it is correlated with the number of autophagosomes, increased LC3 II levels indicate that autophagic activity in the cell is increased $(24,25)$. Even if the role of autophagy in carcinogenesis and tumor progression remains controversial, it has attracted significant attention as a potential anticancer target $(26,27)$.

In the present study, Eca-109 cells were cultured in EBSS medium to induce autophagosome formation and accumulation. Herein, LC3 II/LC3 I was used as a surrogate marker for autophagy levels in cells (28). We observed that EBSS led to the formation of autophagic vacuoles and a prominent increase in the LC3 II/LC3 I ratio in Eca-109 cells at $12 \mathrm{~h}$ post-EBSS treatment, which were markedly attenuated by pre-treatment with LY294002 and 3-MA. In addition, the apoptosis-inducing effect of IR and the implication of EBSS-induced autophagy were investigated. The results suggested that cell apoptosis is induced by IR in a dose-dependent manner; however, it is prominently attenuated by EBSS-induced autophagy, indicating that EBSS-induced autophagy in Eca-109 cells may play a role as a cytoprotective mechanism, in consistence with previously reported results (11).

Cancer cells escape death using a variety of means, with prevention of apoptosis through loss of p53 in some cell types being a classic example $(29,30)$. p53 was recently found to be involved in autophagy regulation (31-33). In the development of pancreatic cancer, p53 may play a key role in blocking tumor progression and promoting cell death and senescence caused by inhibition of autophagy (34). In the present study, increased expression of p53 was observed with EBSS treatment and was markedly attenuated by autophagy inhibition. 
A

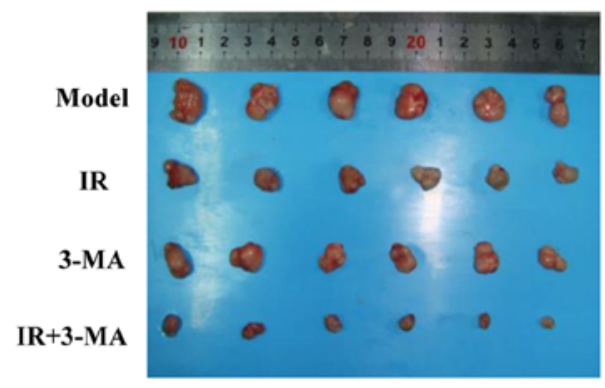

B

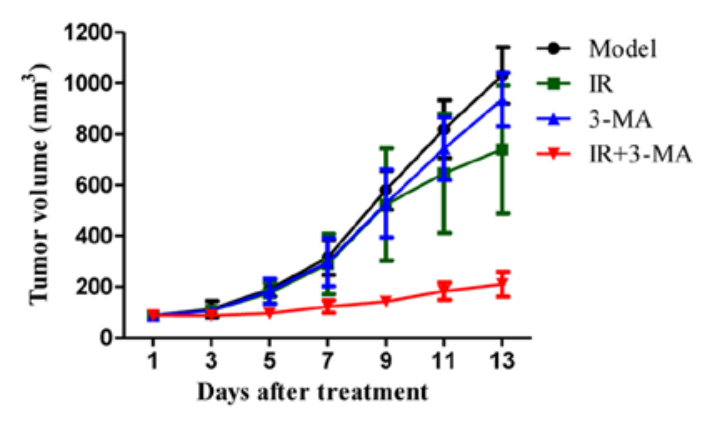

C

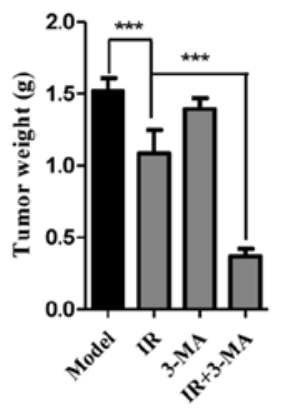

D

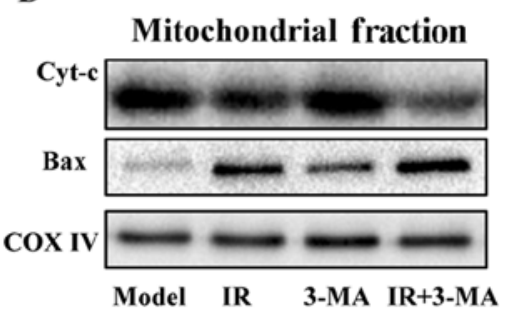

E

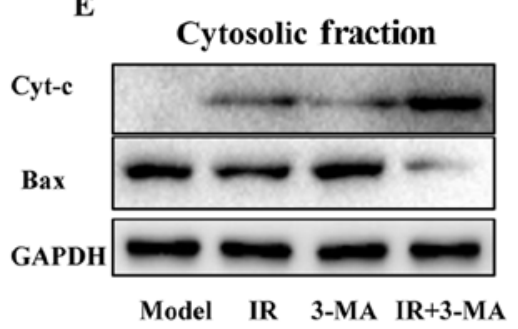

F

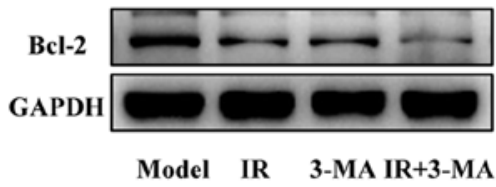

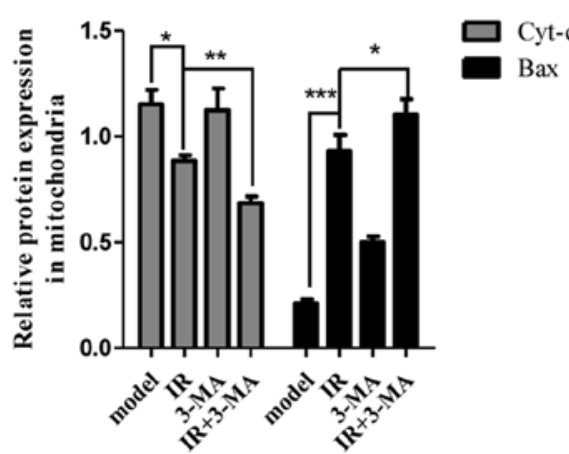
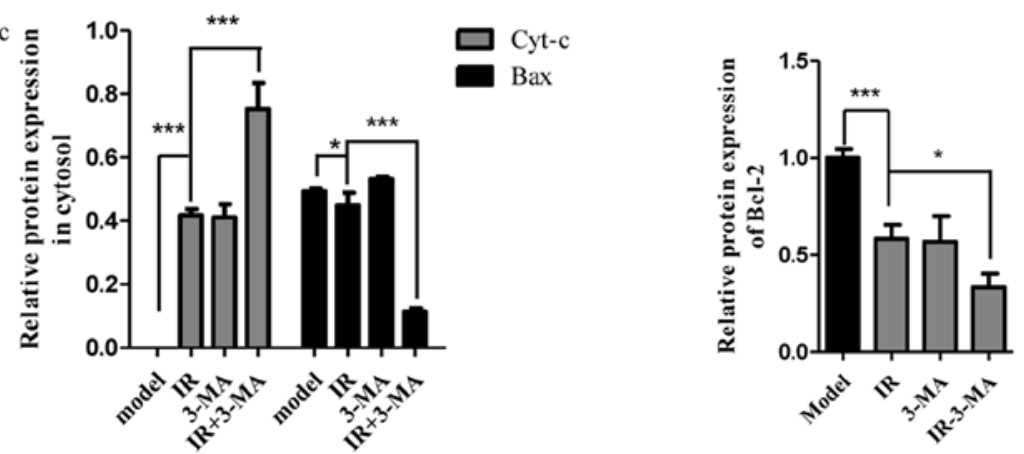

Figure 7. Autophagy inhibition markedly increased the sensitivity of the tumor to IR in vivo. (A-C) Representative images of tumors and tumor growth curve after cell inoculation and treatment with a combination of IR and 3-MA in an Eca-109 nude mouse xenograft model (mean \pm standard deviation, $\mathrm{n}=6$, $\mathrm{P}<0.05$, ${ }^{* *} \mathrm{P}<0.01$ and ${ }^{* * *} \mathrm{P}<0.001$ ). (D-F) The apoptotic proteins Bax, Bcl-2, and cytochrome $c$ in xenografts were analyzed by western blotting and the results of densitometric quantification are shown using GAPDH or COX IV for normalization (mean \pm standard deviation, $\mathrm{n}=3,{ }^{*} \mathrm{P}<0.05,{ }^{* *} \mathrm{P}<0.01$ and ${ }^{* * * *} \mathrm{P}<0.001$ ). COX, cytochrome oxidase; IR, ionizing radiation; 3-MA, 3-methyladenine.

In view of the following results indicating that cell apoptosis was promoted by the combinatorial use of IR and autophagy inhibitors, suppressing the tumor suppressor p53 may provide a possible explanation as to why autophagy inhibition improves the radiosensitivity of Eca-109 cells.

Autophagy inhibition in the present study was performed by administration of LY294002 and 3-MA, and the effect of these autophagy inhibitors on apoptosis and autophagy of starved Eca-109 cells was investigated. The results demonstrated that LY294002 and 3-MA effectively inhibited autophagy without inducing extensive apoptosis at certain concentrations. To distinguish between the apoptosis evoked by IR and that induced by autophagy inhibitors and produce more reliable results, the optimal concentrations of LY294002 and 3-MA were used in the following experiments. Autophagy inhibition combined with IR exhibited possible anticancer value, which has been reported in previous studies $(8,13,35)$. In the present study, we also observed that the radiosensitivity of
Eca-109 cells was significantly enhanced following treatment with LY294002 and 3-MA, which was indicated by increased apoptotic cell numbers and Beclin-1 expression, together with an increased LC3 II/LC3 I ratio. Of note, tumor progression was markedly inhibited in the xenograft nude mouse model following combined treatment with autophagy inhibition and IR. Chen et al used the esophageal carcinoma cell line EC9706 to investigate whether combining radiation with autophagy inhibition enhances the suppression of tumor growth and angiogenesis in esophageal cancer, and the results support our findings (12), suggesting that this may be a common phenomenon in esophageal carcinoma.

To summarize, our results further validated the vital role of autophagy inhibition in radiotherapy for esophageal cancer. Mitochondria are vulnerable to various stimuli and are crucial for regulating cell survival or death. Since a number of studies support that mitochondria are closely associated with autophagy and cancer cell death $\approx(16,36-38)$, it is reasonable to 
hypothesize that the mitochondrial pathway may contribute to enhanced radiosensitivity by autophagy inhibition in esophageal SCC cells. The function of the mitochondrial pathway was investigated in vitro as well as in vivo. It is well known that mitochondrial apoptosis is controlled by proteins of the Bcl-2 family, categorized as antiapoptotic proteins, BH3-only proteins and effectors $(39,40)$. In the mitochondrial pathway, mitochondrial outer membrane permeabilization (MOMP) is the defining event. MOMP may be initiated directly by activating Bax or Bak. Subsequently, cytochrome $c$ and other factors are released and interact with several cytosolic proteins, such as APAF-1, to evoke the activation of caspase. Pro-caspase- 9 is recruited and activated by apoptosome formation and, in turn, the executioners caspase- 3 and -7 are cleaved and activated. Subsequently, the activity of caspase- 3 and -7 kills cells within minutes by cleaving abundant substrates. Even in the absence of caspase activation, post-MOMP mitochondria can hardly maintain cell survival due to their inability to generate ATP $(15,41,42)$. The mechanisms that cancer cells utilize to inhibit mitochondrial apoptosis may be classified as those inhibiting mitochondrial permeabilization and those blocking caspase function (14). After IR, elevated expression of activated caspases and Bax and decreased levels of Bcl-2 were observed in starved Eca-109 cells, along with stimulated release of cytochrome $c$ and decreased MOMP. In addition, application of autophagy inhibitors amplified the changes in mitochondrial pathway-related proteins and MOMP. The role of the mitochondria pathway was further studied in an Eca-109 xenograft nude mouse model, the findings of which were consistent with those of the in vitro experiments.

In conclusion, the results of the present study demonstrated that autophagy inhibition markedly enhanced the cytotoxicity of IR and suppressed tumor growth through the mitochondrial pathway. Our results further supported the value of autophagy inhibitors in the radiotherapy of esophageal SCC, and the significance of mitochondria in cancer radiotherapy.

\section{Acknowledgements}

The authors would like to thank Yuan Liu, Xi Yu, Lei Ao and Lei Cao for providing experimental advice and technical help.

\section{Funding}

The present study was supported by a grant from the Jiangsu Cancer Hospital (no. ZM201207).

\section{Ethics approval and consent to participate}

All experiments involving mice were performed in conformity with the guidelines on animal care and experiments on laboratory animals of the Center of Experimental Animals, Nanjing University of Technology (Nanjing, China), and were approved by the Ethics Committee for Animal Experimentation (no. OGKQSPF/SQ-03).

\section{Availability of data and materials}

The analyzed data sets generated during the study are available from the corresponding author on reasonable request.

\section{Authors' contributions}

HT wrote the manuscript. HT, PDQ and FJW designed the experiments, HT, PDQ, JCL, YSG and HFZ performed experiments and analysed the data. The final version of the manuscript has been read and approved by all authors.

\section{Consent for publication}

Not applicable.

\section{Competing interests}

The authors declare that they have no competing interests.

\section{References}

1. Ferlay J, Shin HR, Bray F, Forman D, Mathers C and Parkin DM: Estimates of worldwide burden of cancer in 2008: GLOBOCAN 2008. Int J Cancer 127: 2893-2917, 2010.

2. Jemal A, Siegel R, Ward E, Hao Y, Xu J and Thun MJ: Cancer statistics, 2009. CA Cancer J Clin 59: 225-249, 2009.

3 . Enzinger PC and Mayer RJ: Esophageal cancer. N Engl J Med 349: 2241-2252, 2003

4. Kamangar F, Dores GM and Anderson WF: Patterns of cancer incidence, mortality, and prevalence across five continents: Defining priorities to reduce cancer disparities in different geographic regions of the world. J Clin Oncol 24: 2137-2150, 2006.

5. Eslick GD: Epidemiology of esophageal cancer. Gastroenterol Clin North Am 38: 17-25, 2009.

6. Murrow L and Debnath J: Autophagy as a stress-response and quality-control mechanism: Implications for cell injury and human disease. Annu Rev Pathol 8: 105-137, 2013.

7. Martinet W, De Meyer GR, Andries L, Herman AG and Kockx MM: In situ detection of starvation-induced autophagy. J Histochem Cytochem 54: 85-96, 2006.

8. Yang Y, Yang Y, Yang X, Zhu H, Guo Q, Chen X, Zhang H, Cheng $\mathrm{H}$ and Sun $\mathrm{X}$ : Autophagy and its function in radiosensitivity. Tumour Biol 36: 4079-4087, 2015.

9. Chen X, Wang P, Guo F, Wang X, Wang J, Xu J, Yuan D, Zhang $J$ and Shao C: Autophagy enhanced the radioresistance of non-small cell lung cancer by regulating ROS level under hypoxia condition. Int J Radiat Biol 93: 764-770, 2017.

10. Lomonaco SL, Finniss S, Xiang C, Decarvalho A, Umansky F, Kalkanis SN, Mikkelsen T and Brodie C: The induction of autophagy by gamma-radiation contributes to the radioresistance of glioma stem cells. Int J Cancer 125: 717-722, 2009.

11. Chaachouay H, Ohneseit P, Toulany M, Kehlbach R, Multhoff G and Rodemann HP: Autophagy contributes to resistance of tumor cells to ionizing radiation. Radiother Oncol 99: 287-292, 2011.

12. Chen Y, Li X, Guo L, Wu X, He C, Zhang S, Xiao Y, Yang Y and Hao D: Combining radiation with autophagy inhibition enhances suppression of tumor growth and angiogenesis in esophageal cancer. Mol Med Rep 12: 1645-1652, 2015.

13. Liang DH, El-Zein R and Dave B: Autophagy inhibition to increase radiosensitization in breast cancer. J Nucl Med Radiat Ther 6: 6, 2015.

14. Lopez J and Tait SW: Mitochondrial apoptosis: Killing cancer using the enemy within. Br J Cancer 112: 957-962, 2015.

15. Tait SW and Green DR: Mitochondria and cell death: Outer membrane permeabilization and beyond. Nat Rev Mol Cell Biol 11: 621-632, 2010.

16. Chen Z, Wang B, Yu F, Chen Q, Tian Y, Ma S and Liu X: The roles of mitochondria in radiation-induced autophagic cell death in cervical cancer cells. Tumour Biol 37: 4083-4091, 2016.

17. Bristol ML, Di X, Beckman MJ, Wilson EN, Henderson SC, Maiti A, Fan Z and Gewirtz DA: Dual functions of autophagy in the response of breast tumor cells to radiation: Cytoprotective autophagy with radiation alone and cytotoxic autophagy in radiosensitization by vitamin D 3. Autophagy 8: 739-753, 2012.

18. Wilson EN, Bristol ML, Di X, Maltese WA, Koterba K, Beckman MJ and Gewirtz DA: A switch between cytoprotective and cytotoxic autophagy in the radiosensitization of breast tumor cells by chloroquine and vitamin D. Horm Cancer 2: 272-285, 2011. 
19. Apel A, Herr I, Schwarz H, Rodemann HP and Mayer A: Blocked autophagy sensitizes resistant carcinoma cells to radiation therapy. Cancer Res 68: 1485-1494, 2008.

20. Kondo Y, Kanzawa T, Sawaya R and Kondo S: The role of autophagy in cancer development and response to therapy. Nat Rev Cancer 5: 726-734, 2005.

21. Sotelo J, Briceño E and López-González MA: Adding chloroquine to conventional treatment for glioblastoma multiforme: A randomized, double-blind, placebo-controlled trial. Ann Intern Med 144: 337-343, 2006.

22. Livak KJ and Schmittgen TD: Analysis of relative gene expression data using real-time quantitative PCR and the 2(-Delta Delta C(T)) Method. Methods 25: 402-408, 2001.

23. Feng Y, He D, Yao Z and Klionsky DJ: The machinery of macroautophagy. Cell Res 24: 24-41, 2014.

24. Mizushima N and Yoshimori T: How to interpret LC3 immunoblotting. Autophagy 3: 542-545, 2007.

25. Kabeya Y, Mizushima N, Ueno T, Yamamoto A, Kirisako T, Noda T, Kominami E, Ohsumi Y and Yoshimori T: LC3, a mammalian homologue of yeast Apg8p, is localized in autophagosome membranes after processing. EMBO J 19: 5720-5728, 2000.

26. Amaravadi RK, Lippincott-Schwartz J, Yin XM, Weiss WA, Takebe N, Timmer W, DiPaola RS, Lotze MT and White E: Principles and current strategies for targeting autophagy for cancer treatment. Clin Cancer Res 17: 654-666, 2011.

27. Grandér D and Panaretakis T: Autophagy: Cancer therapy's friend or foe? Future Med Chem 2: 285-297, 2010

28. He Y, Zhao X, Subahan NR, Fan L, Gao J and Chen H: The prognostic value of autophagy-related markers beclin-1 and microtubule-associated protein light chain 3B in cancers: A systematic review and meta-analysis. Tumour Biol 35: 7317-7326, 2014.

29. Clarke AR, Purdie CA, Harrison DJ, Morris RG, Bird CC, Hooper ML and Wyllie AH: Thymocyte apoptosis induced by p53-dependent and independent pathways. Nature 362: 849-852, 1993.

30. Lowe SW, Schmitt EM, Smith SW, Osborne BA and Jacks T: p53 is required for radiation-induced apoptosis in mouse thymocytes. Nature 362: 847-849, 1993

31. Eisenberg-Lerner A, Bialik S, Simon HU and Kimchi A: Life and death partners: Apoptosis, autophagy and the cross-talk between them. Cell Death Differ 16: 966-975, 2009.

32. Crighton D, Wilkinson S, O'Prey J, Syed N, Smith P, Harrison PR, Gasco M, Garrone O, Crook T and Ryan KM: DRAM, a p53-induced modulator of autophagy, is critical for apoptosis. Cell 126: 121-134, 2006.
33. Maiuri MC,Malik SA,MorselliE,KeppO,Criollo A, Mouchel PL, Carnuccio R and Kroemer G: Stimulation of autophagy by the p53 target gene Sestrin2. Cell Cycle 8: 1571-1576, 2009.

34. Rosenfeldt MT, O'Prey J, Morton JP, Nixon C, MacKay G, Mrowinska A, Au A, Rai TS, Zheng L, Ridgway R, et al: p53 status determines the role of autophagy in pancreatic tumour development. Nature 504: 296-300, 2013.

35. Han MW, Lee JC, Choi JY, Kim GC, Chang HW, Nam HY, Kim SW and Kim SY: Autophagy inhibition can overcome radioresistance in breast cancer cells through suppression of TAK1 activation. Anticancer Res 34: 1449-1455, 2014.

36. Chen Z, Liu X and Ma S: The roles of mitochondria in autophagic cell death. Cancer Biother Radiopharm 31: 269-276, 2016.

37. Qiao ZY, Lai WJ, Lin YX, Li D, Nan XH, Wang Y, Wang H and Fang QJ: Polymer-KLAK peptide conjugates induce cancer cell death through synergistic effects of mitochondria damage and autophagy blockage. Bioconjug Chem 28: 1709-1721, 2017.

38. Danese A, Patergnani S, Bonora M, Wieckowski MR, Previati M, Giorgi $\mathrm{C}$ and Pinton P: Calcium regulates cell death in cancer: Roles of the mitochondria and mitochondria-associated membranes (MAMs). Biochim Biophys Acta 1858: 615-627, 2017.

39. Kim H, Rafiuddin-Shah M, Tu HC, Jeffers JR, Zambetti GP Hsieh JJ and Cheng EH: Hierarchical regulation of mitochondrion-dependent apoptosis by BCL-2 subfamilies. Nat Cell Biol 8: 1348-1358, 2006.

40. Kim H, Tu HC, Ren D, Takeuchi O, Jeffers JR, Zambetti GP, Hsieh JJ and Cheng EH: Stepwise activation of BAX and BAK by tBID, BIM, and PUMA initiates mitochondrial apoptosis. Mol Cell 36: 487-499, 2009.

41. Colell A, Ricci JE, Tait S, Milasta S, Maurer U, Bouchier-Hayes L, Fitzgerald P, Guio-Carrion A, Waterhouse NJ, Li CW, et al: GAPDH and autophagy preserve survival after apoptotic cytochrome $c$ release in the absence of caspase activation. Cell 129: 983-997, 2007.

42. Lartigue L, Kushnareva Y, Seong Y, Lin H, Faustin B and Newmeyer DD: Caspase-independent mitochondrial cell death results from loss of respiration, not cytotoxic protein release. Mol Biol Cell 20: 4871-4884, 2009.

This work is licensed under a Creative Commons Attribution-NonCommercial-NoDerivatives 4.0 International (CC BY-NC-ND 4.0) License. 